\title{
Fusion energy using avalanche increased boron reactions for block-ignition by ultrahigh power picosecond laser pulses
}

\author{
HEINRICH HORA, ${ }^{1}$ GEORG KORN,${ }^{2}$ LORENZO GIUFFRIDA, ${ }^{2}$ DANIELE MARGARONE, ${ }^{2}$ \\ ANTONINO PICCIOTTO, ${ }^{3, *}$ JOSEF KRASA, ${ }^{2}$ KAREL JUNGWIRTH,${ }^{4}$ JIRI ULLSCHMIED, ${ }^{4}$ \\ PARASKEVAS LALOUSIS, ${ }^{5}$ SHALOM ELIEZER,,${ }^{6,7}$ GEORGE H. MILEY, ${ }^{8}$ STAVROS MOUSTAIZIS, ${ }^{9}$ \\ AND GÉRARD MOUROU ${ }^{10}$ \\ ${ }^{1}$ Department of Theoretical Physics, University of New South Wales, Sydney, Australia \\ ${ }^{2}$ Institute of Physics ASCR, v.v.i (FZU), ELI-Beamlines project, Prague, Czech Republic \\ ${ }^{3}$ Micro-Nano Facility, Fondazione Bruno Kessler, Trento, Italy \\ ${ }^{4}$ PALS Laboratory, Institute of Plasma Physics of the ASCR, Prague, Czech Republic \\ ${ }^{5}$ Institute of Electronic Structure and Lasers FORTH, Heraklion, Greece \\ ${ }^{6}$ Institute of Nuclear Fusion, Polytechnic University of Madrid, Madrid, Spain \\ ${ }^{7}$ Soreq Research Center, Yavne, Israel \\ ${ }^{8}$ Department Nuclear Plasma \& Radiological Engineering, University of Illinois, Urbana, Illinois \\ ${ }^{9}$ Laboratory of Matter Structure and Laser Physics, Technical University of Crete, Chania, Greece \\ ${ }^{10}$ DAER-IZEST, Ecole polytechnique, Route de Saclay, Palaiseau Cedex, France \\ (RECEIVED 9 May 2015; ACCEPTED 8 June 2015)
}

\begin{abstract}
Exceptionally high reaction gains of hydrogen protons measured with the boron isotope 11 are compared with other fusion reactions. This is leading to the conclusion that secondary avalanche reactions are happening and confirming the results of high-gain, neutron-free, clean, safe, low-cost, and long-term available energy. The essential basis is the unusual nonthermal block-ignition scheme with picosecond laser pulses of extremely high powers above the petawatt range.
\end{abstract}

Keywords: Fusion energy without radiation problem; Boron fusion by lasers; Non-linear force-driven block ignition; Ultrahigh acceleration; Ultrahigh magnetic fields

\section{INTRODUCTION}

Fusion reactions of hydrogen protons with the boron isotope 11 (HB11) (Oliphant \& Rutherford, 1933) were from the beginning, of special interest. These occurred at collision energies of or below $100 \mathrm{keV}$, while nuclear reactions due to the Coulomb barrier needed always several $\mathrm{MeV}$ per proton to squeeze the nuclei to distances of their diameters of femtometers (fm). This was the first time to measure the nuclear reactions occurring at distances in the range of $100 \mathrm{fm}$ and lead to the discovery of the reaction of heavy hydrogen, deuterium $\mathrm{D}$, with the super heavy hydrogen tritium $\mathrm{T}$ (Oliphant et al., 1934). That these reactions occurred at 100 times higher distance showed that the DT fusion reaction was extremely exceptional occurring at collision energies of several keV.

Address correspondence and reprint requests to: Heinrich Hora, Department of Theoretical Physics, University of New South Wales, Sydney 2052, Australia. E-mail: h.hora@unsw.edu.au

*Author's name has been corrected since original publication. An erratum notice detailing this change was also published (DOI 10.1017/ S0263034615000750).
DT is the easiest binary nuclear reaction and preferably used to study energy production in controlled nuclear fusion power generators

$$
\mathrm{D}+\mathrm{T}={ }^{4} \mathrm{He}+\mathrm{n}+17.6 \mathrm{MeV}
$$

resulting in clean helium $(\mathrm{He})$ and in neutrons $\mathrm{n}$ of $14.1 \mathrm{MeV}$ energy and a lifetime $12.6 \mathrm{~min}$ before decaying into an electron and a proton. During this lifetime the neutrons can penetrate into clean (non-radioactive, stable) nuclei and change them into dangerous radioactive nuclei. One has to realize that DT fusion primarily produces nearly four times more neutrons per gained energy than in fission reactors and another difference is that the transmuted stable nuclei in a fission reactor (referring here to the completely established fission power stations or the advancement of small modular reactors (Paterson, 2015) by Westinghouse or Chinese-Australian cooperation) are mostly contained in the nuclear waste and much easier kept under control than in fusion reactors where they are spreading through the blanket and escape to 
transmute widely dispersed clean stable nuclei into dangerous ones. This fact was described as disadvantage that DT fusion leads to the "hottest radioactive environment on earth" which was explicitly endorsed by Sir William Mitchell (Butler, 1996). It was elaborated how this problem of DT fusion has to be overcome (Tahir \& Hoffmann, 1997). However, the following reported new aspects may lead to other options for fusion energy; it has to be acknowledged that these all would not have been reached than by standing on the shoulders of the long past and ongoing most extensive research and achievements gained from fusion work with DT.

The other important result of the HB11 fusion (Oliphant \& Rutherford, 1933) is the generation of mostly equal energetic He nuclei (a-particles)

$$
\mathrm{H}+{ }^{11} \mathrm{~B}=3^{4} \mathrm{He}+8.7 \mathrm{MeV} .
$$

Apart from the result of the anomalous large distant reaction, the fact is important that no neutrons are being generated primarily. This was discussed (Mitchell, 2000) also in contacts with J.C. Kelly (Hora et al., 2009). It was clarified (Weaver et al., 1973) that any side reactions produce less radioactivity per gained energy than by burning coal due to its contents of $2 \mathrm{ppm}$ uranium. Therefore, any nuclear radiation problem of HB11 fusion can be ignored.

The only earlier problem before the present new developments was the fact that the HB11 fusion was extremely more difficult than DT fusion if the usual gas-dynamic compressions and thermal ignition with laser pulses of nanosecond (ns) duration were applied. Even if the advanced measurements of the fusion cross-sections at the resonance energy of $148 \mathrm{keV}$ (Nevins et al., 2000) were used (Kouhi et al., 2011), the compression of the HB11 fuel had to be above 100,000 times of the solid state. The more it was surprising when instead of the ns laser pulses with their thermal ignition of the fusion, an alternative non-thermal block ignition with picosecond (ps) laser pulses of extreme high power was used in the computations (Hora et al., 2010), that then the difficulty for HB11 laser fusion had changed and drastically decreased to about the same limits as for DT.

This enormous improvement, however, was not sufficient for reaching conditions of economic energy production with HB11 laser fusion. Up to this stage (Hora et al., 2010; Lalousis et al., 2013), the computations were still pessimistically using only binary reactions as always applied for fusion. The necessary further improvement beyond the binary reactions for HB11, however, is essential for the favorable nonthermal ps-block ignition described in the following. The experimental results for this improvement are by secondary reactions after the binary reactions which are first described in the following section before the later sections are applying this result to the details of HB11 block ignition.

\section{VERY HIGH BORON FUSION EXPLAINED AS AVALANCHE REACTION}

The strong interest in the HB11 fusion reaction was evident and a large number of proposals were considered including extreme non-thermal magnetic field configurations in lowdensity plasmas (Rostoker et al., 1997). Substantial progress was reached when direct measurements of the reaction were performed (Belyaev et al., 2005). This found attention though the laser-driven plasmas produced only 1000 reactions, just close to the level of experimental sensitivity. A convincing and very transparent result (Labaune et al., 2013) produced more than $10^{6}$ reactions though only a complicated combination of laser-driven directed particle beams with a secondary laser-target interaction was used. After this breakthrough, the measurement of $10^{9} /$ sr reactions (Picciotto et al., 2014; Margarone et al., 2015) was achieved. These measurements were a straightforward laser-fusion result by a unique combination of one of the largest European lasers, the PALS iodine laser at Prague, with the use of a very unusual laser-irradiated target. However, these fusion gains were too high compared with preceding knowledge.

The iodine laser matured (Hohla \& Kompa, 1973) to a precisely working system only after a fast inductive operation for the photo-ionizing flash lamps was possible and a high technology amplifier system was developed (Witte, 1990, private communication) for operation in an optimized environment of PALS (Jungwirth et al., 2001). Laser pulses in the range of 100 ps duration and up to kJ energy provided a system which was uniquely different from the broad stream of development.

The other unusual ingredient of the experiment (Picciotto et al., 2014; Margarone et al., 2015) was the use of an exotic target, consisting of silicon with an extremely high concentration of boron. Low concentration of boron produced the transistor effects from the beginning and the very high concentration is in the focus of semiconductor research for degenerate hole concentration. This is a crossing point of solid-state physics with high laser intensity interaction showing an influence by surface effects due to high trap concentrations (Boreham et al., 1995). It was the special advantage to combine the solid-state physics research of silicon with extremely high boron concentration (apart from the natural high proton concentration) for the HB11 laser fusion experiments at PALS.

The result (Picciotto et al., 2014) was most significant that a PALS laser pulse of $200 \mathrm{~J}$ on the special silicon target resulted in the generation of $10^{9} / \mathrm{sr} \mathrm{HB} 11$ reactions. This was considerable more than 100 times higher than measured before (Labaune et al., 2013), with the further advantage that the experiment (Picciotto et al., 2014) was a direct and transparent laser-fusion experiment without any complex modification due to ion beams, etc.

After presenting these results with extraordinary high enhancement of the yield of induced nuclear reacting ultraclean boron fusion (Korn et al., 2014), the evaluation was followed 
up how these gains could have happened. The experiment was within the category of ns laser pulse-driven fusion without special conditions of pulse contrast preparation for suppression of prepulses, etc. Considering the neutron gains $N$ from DT reactions depending on the energy $E_{\mathrm{p}}$ of ns laser pulses in Figure 1 (Hora, 2013a), an approximate relation

$$
N=\text { const } E_{\mathrm{p}}^{2}
$$

can be derived. Comparing the gain of $10^{9}$ for $200 \mathrm{~J}$ laser pulses with the 1.9 MJ pulse of the highest measured DT reaction would correspond to $3.6 \times 10^{17}$ reactions which is far above the measured value (Hurricane et al., 2014). This permits the conclusion that the evaluation of the HB11 reaction (Korn et al., 2014) cannot be a binary reaction as in usual computations for fusion.

The conclusion is that the measured strongly enhanced reaction yield of HB11 is the result of secondary reactions as was discussed before (Hora et al., 2012a) mentioned as "secondary reactions" (Folgereaktionen) in a patent application (Hora, 2013b) causing an avalanche increase of the reaction. Permission for no restrictions at publications was given formally by the German Patent Office (Hora, 2014) on which documentation the open discussion is based (Hora et al., $2014 b$ ). This can be seen from considering elastic central collisions between the mass $m_{\mathrm{a}}$ of an a-particle of energy $E_{\mathrm{a}}$ with an initially resting ${ }^{11} \mathrm{~B}$ nucleus with the mass $m_{\mathrm{B}}$. The energy of the boron after collision is (Hora et al., 2012a)

$$
E_{\mathrm{B}}=4 m_{\mathrm{a}} m_{\mathrm{B}} E_{\mathrm{a}} /\left(m_{\mathrm{a}}+m_{\mathrm{B}}\right)^{2}
$$

Using the initial energy of the a-particle from the HB11 reaction of $2.9 \mathrm{MeV}$, the first elastic hit transfers $530 \mathrm{keV}$ energy from the $\alpha$-particle to the boron. After this collision, the $\alpha$-particle can produce a second hit to boron which is then

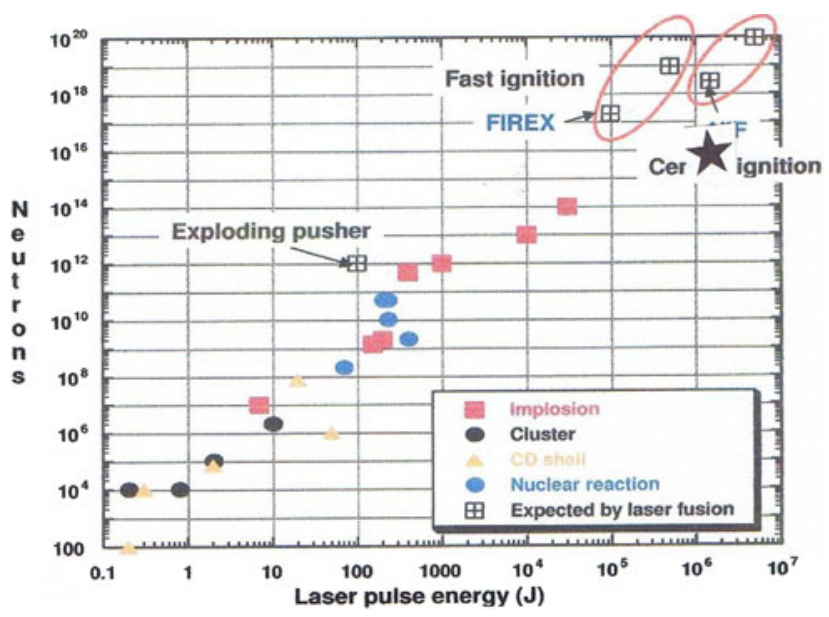

Fig. 1. Measured highest DT fusion neutron gains $N$ per incident laser energy (full signs) of laser pulses in the range of ns duration (Hora, $2013 a$ ) with the highest value reported (Hurricane et al., 2014) given by an asterisk. gaining $492 \mathrm{keV}$, etc. As known from Nevins et al. (2000), there is an exceptional high fusion cross-section, widely spread, with protons at $550 \mathrm{keV}$ energy, about ten times higher than known from other fusion reactions. The primary produced $\alpha$-particles are then causing a secondary reaction resulting in three new alphas, etc., if there is not a problem with the stopping length of the alphas.

Instead of studying several stopping power models using appropriate simplifications, the discussion of the measured strongly enhanced reaction yield of HB11 (Korn et al., 2014; Hora et al., 2015) can be used as clarification that there is the avalanche process happening. This avalanche process for a fusion reaction is unique for HB11 in contrast to the other fusion reactions which are based only on binary reactions without the possibility of a following-up reaction.

As a further support for the used relation of Eq. (3) for DT, the reaction of DD for the ns range of laser pulses shows a similar relation (Krasa et al., 2013) only with the well-known lower reaction gain by a factor of about 300 . These results for the longer laser pulses are to be separated from results with ps pulses for DD fusion. In these cases, the DD gains can be higher than the normal relation of the kind of Eq. (3) with the increase of more than $10^{4}$ reported by Norreys et al. (1998) based on extremely clean laser pulses with high contrast ratio. This case is, the first example of a fusion experiment showing the drastic difference between thermal interactions with ns pulses in contrast to the non-thermal direct ps transfer of optical laser energy into macroscopic motion of plasma blocks considered in the following sections (Hora, 2003).

\section{NON-THERMAL LASER ENERGY TRANSFER TO TARGETS}

In order to explain the laser fusion mechanism on which the following results with application of the just reported avalanche reaction for HB11 is based, we summarize the main achievements of the plasma block initiation of the ignition process based on ps laser pulses. This is basically different from the mostly studied laser fusion process with ns laser pulses using gas-dynamic thermal heating, pressurecompression, and ignition processes with complex thermalizing optical absorption, electron-ion energy-delayed classical or quantum defined ion-heating mechanisms (Hora et al., $2012 b$ ), including instabilities, radiation losses, thermally determined reactions, etc. The details of these processes are now mostly under control (Hurricane et al., 2014), while different options of the direct or indirect drive, volume or spark ignition and self-heat by the reaction process, and reabsorption of bremsstrahlung are involved (Hora, 2013a).

For understanding the basic difference of the non-thermal and to some extent simplified plasma block-ignition process based on non-linear (ponderomotive-dielectric determined) forces using ps laser pulses, we have to remind to the dozens of years lasting development. When the early laser 
pulses of frequency $\omega$ with powers below MW were focused on targets, the heating and dynamic processes followed all the preceding known physics with emission of ions of few $\mathrm{eV}$ according to temperatures of about $30,000 \mathrm{~K}$. But at only 10 times higher laser power, ions had 1000 times higher energies and were separated by their charge numbers confirming non-thermal, electrodynamic interaction. This led to the derivation of forces by lasers in media with spatial or temporal variation of dielectric properties (Hora, 1969; 1985) using the refractive index $\mathbf{n}$ of the generated plasmas. The force density was then not only given by the gas-dynamic pressure $p$

$$
\mathbf{f}=-\nabla p+\mathbf{f}_{\mathrm{NL}}
$$

but also by Maxwell's stress tensor as Lorentz and gaugeinvariant non-linear force (Hora, 1969; 1985; 2000)

$$
\begin{aligned}
\mathbf{f}_{\mathrm{NL}}= & \nabla \bullet\left[\mathbf{E E}+\mathbf{H H}-0.5\left(\mathbf{E}^{2}+\mathbf{H}^{2}\right) \mathbf{1}+(1+(\partial / \partial t) / \omega)\right. \\
& \left.\times\left(\mathbf{n}^{2}-1\right) \mathbf{E E}\right] /(4 \pi)-(\partial / \partial t) \mathbf{E} \times \mathbf{H} /(4 \pi c) .
\end{aligned}
$$

At plane laser wave interaction with a plane plasma front, the force reduces to

$$
\begin{aligned}
\mathbf{f}_{\mathrm{NL}} & =-(\partial / \partial x)\left(\mathbf{E}^{2}+\mathbf{H}^{2}\right) /(8 \pi) \\
& =-\left(\omega_{\mathrm{p}} / \omega\right)^{2}(\partial / \partial x)\left(E_{\mathrm{v}}^{2} / \mathbf{n}\right) /(16 \pi)
\end{aligned}
$$

showing how the force density is given by the negative gradient of the electromagnetic laser field density. Expressing the magnetic laser field from Maxwell's equations by the electric field results in the final expression which is equal to Kelvin's ponderomotive force in electrostatics of 1845 . For non-linear correct results - in contrast to incorrect linear physics, see Feynman, Section 6.3 of Hora (2000) all components of the stress tensor have to be used Cicchitelli (1990).

Following the very first general hydrodynamic computations with the force (5), the plasma motion at sufficiently high laser intensities is dominated by the non-linear force well with some corrections by the thermodynamic processes (Hora et al., 1978). Drawn are the results for $10^{18} \mathrm{~W} / \mathrm{cm}^{2}$ laser intensity from (Hora, 1981: Fig. 10.18a \& b) at plane geometry irradiation in Figure 2 incident from the right-hand side on a deuterium plasma with an initial bi-Rayleigh density profile (Fig. 10.17 of Hora, 1981). This results in a plasma block of more than 15 vacuum wavelengths thickness moving after $1.5 \mathrm{ps}$ against the laser and a deeper block inside the deuterium parallel to the laser direction into the interior of the deuterium. The acceleration of each of the plasma blocks is above $10^{20} \mathrm{~cm} / \mathrm{s}^{2}$ for reaching velocities above $10^{9} \mathrm{~cm} / \mathrm{s}$ during $1.5 \mathrm{ps}$.

This ultrahigh acceleration was measured for the plane geometry by Sauerbrey (1996) which was 100,000 times higher than ever measured before in a laboratory. The sophisticated measurements by Doppler shift of spectral lines reflected

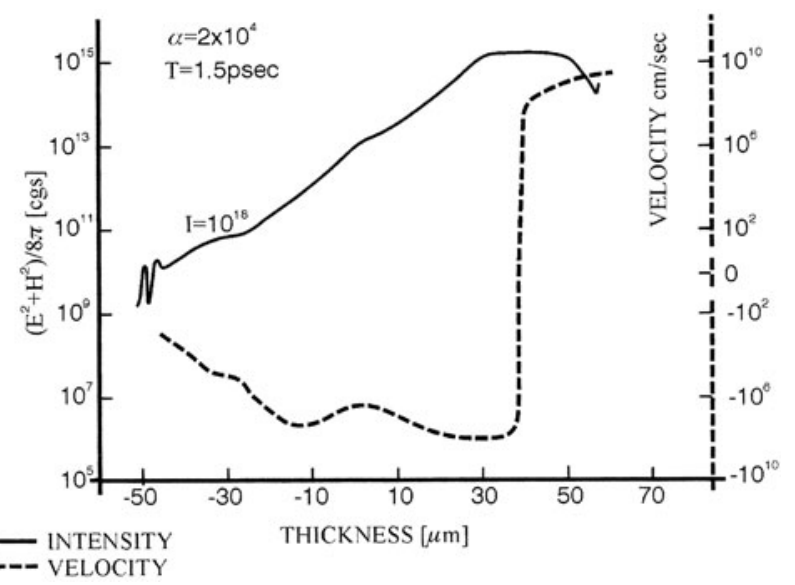

Fig. 2. $10^{18} \mathrm{~W} / \mathrm{cm}^{2}$ neodymium glass laser incident from the right-hand side on an initially $100 \mathrm{eV}$ hot deuterium plasma whose density has a very low-reflecting bi-Rayleigh profile at initial time $t=1$, resulting in a laser energy density and a velocity distribution from plasma hydrodynamic computations at an interaction time $t=1.5 \mathrm{ps}$ of interaction. The driving nonlinear force is the negative of the varying energy density of the laser field $\left(E^{2}+H^{2}\right) / 8 \pi$ expressing the intensity. The dynamic development of temperature and density had accelerated the plasma block of about 20 vacuum wavelength thickness of the dielectric enlarged skin layer moving against the laser (positive velocity) and another block into the plasma (negative velocity) showing ultrahigh $>10^{20} \mathrm{~cm} / \mathrm{s}^{2}$ acceleration.

from the outer block moving against the incident laser beam were repeated by Földes et al. (2000) in agreement with the theory of non-linear force acceleration evaluated (Hora et al., 2002a; 2007). The ion density in the space charge neutral blocks was - as theoretically predicted 1978 above $10^{12} \mathrm{Amp} / \mathrm{cm}^{2}$ (Hora et al., 2002b).

The generation of the ultrahigh acceleration of the nonlinear force-driven plasma blocks was considered from the beginning for the explanation of the measured highly directed plasma motion with the high ion energies (Hora, 1981) as shown in Figure 2 with the plasma block moving to the righthand side against the laser light and the other block moving into the interior of the target. Schematically, this can be seen in Figure 3 (Hora et al., 2002b). Apart from the appearance of the ultrahigh velocities, the high densities of the space charge neutral plasma blocks resulted in very high ion current densities above $10^{12} \mathrm{Amp} / \mathrm{cm}^{2}$ (Hora et al., 2002a) which values were predicted in the computer outputs of 1978 (Hora et al., 1978; Hora, 1981) (Fig. 2). These ion current densities are more than million times higher than in conventional accelerators. A repetition of the experiments by Sauerbrey (1996) was possible by Földes et al. (2000), Figure 4, using the appropriately lower laser intensities in accurate agreement with the theory.

The ultrahigh acceleration was comparably similar when treated by Target Normal Sheath Acceleration (TNSA) (Yu et al., 2005), calling then the blocks "bunch" with more details of the relatively much higher Debye length than in Figure 3. The cases of Yu et al. (2005) were chosen for the lower target thickness and other plasma parameters resulting 


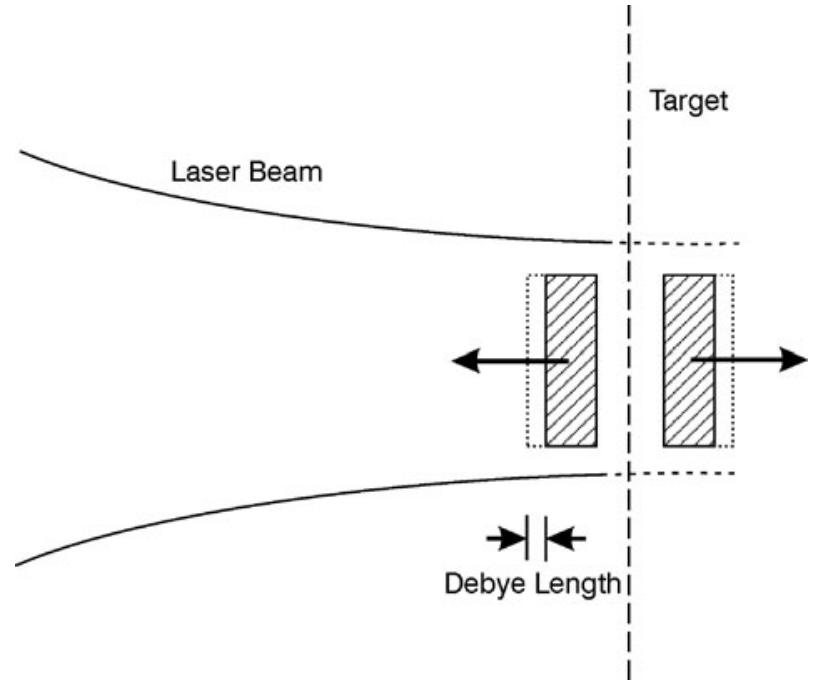

Fig. 3. Scheme of skin depth laser interaction, where the non-linear force accelerates a plasma block against the laser light and another block toward the target interior. In front of the blocks are electron clouds of the thickness of the effective Debye lengths [Eq. (5)] (Hora et al., 2002b).

then, in a more pronounced sheath generation which could be seen also from a double-layer model (Eliezer et al., 2014) arriving at corresponding results.

The most important need for the discovery to confirm the theoretical prediction of the ultrahigh acceleration (Sauerbrey, 1996; Földes et al., 2000) and of the ultrahigh ion current densities (Hora et al., 2002a) was based on Mourou's et al., chirped-pulse amplification (CPA) to generate extreme powerful laser pulses of ps and shorter duration reaching now 7.12 petawatt (PW) power and 27 fs duration (Li et al., 2015) with Ti:sapphire lasers with sufficient suppression of transverse parasitic lasing have been produced with the aim that 30 PW-ps pulses may be available within few years by combining of a number of beams, if not fiber lasers with singlemode (Mourou et al., 2013) and spherically focused (Hora et al., 2014a) output will be available. This was crucial for the measurement by Sauerbrey (1996) together with the second

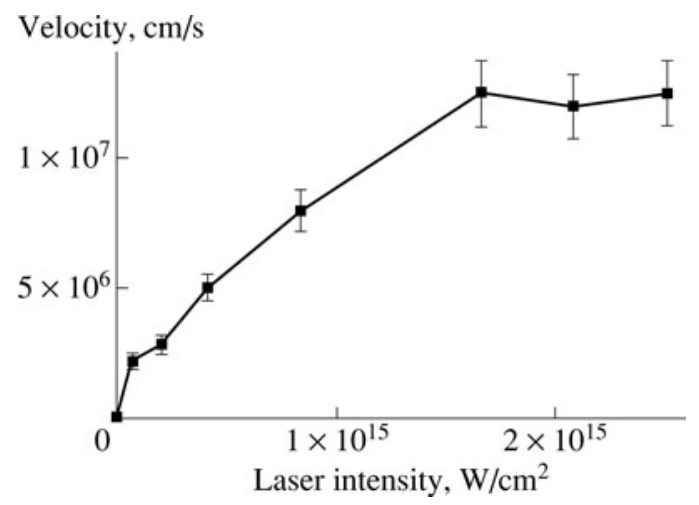

Fig. 4. Intensity dependence of the velocity of the plasma front from the Doppler line shift of the reflected from the irradiation of $700 \mathrm{fs} \mathrm{KrF}$ laser pulses on an aluminum target (Földes et al., 2000). ingredient of using an extreme contrast ratio against prepulses to avoid relativistic self-focusing as clarified by Zhang et al. (1998).

It should be underlined that the block ignition can be considered as a modification of the fast ignition as introduced in 1991 by Campbell (2006) and elaborated by Tabak et al. (1994).

\section{BLOCK IGNITION FOR LASER FUSION}

The non-thermal generation of plasma blocks by ultrahigh acceleration with ps laser pulses by the non-linear force can be used for the ignition of fusion following the discovery by Chu (1972) and Bobin (1974) for initiation of a fusion reaction flame to ignite solid density DT fuel. The result was, however, that the ps laser pulses have at least a minimum energy flux density of few $E^{*}=10^{8} \mathrm{~J} / \mathrm{cm}^{2}$. Updating the theory (Hora et al., 2008) led to a reduction of $E^{*}$ by up to a factor 20 .

First we report block-ignited fusion results for DT fusion before continuing about HB11. The ps pulse-generated plasma blocks with their ultrahigh acceleration initiated a fusion flame in solid density DT where plane wave laser interaction with an energy flux density $E^{*}$ of $10^{8} \mathrm{~J} / \mathrm{cm}^{2}$ was necessary (Chu, 1972; Bobin, 1974). The updating of the computations of Chu (1972) arrived at similar results (Hora, 2009). These computations used one-fluid hydrodynamics results where the updating was necessary for the later known reduction of the thermal conduction by an inhibition factor based on the electric double layer between the hot flame and the DT fuel. Further, the stopping power had to use the Gabor collective model at this high plasma densities and not the Bethe-Bloch binary collision model. After this updating, the ignition thresholds for the fusion flame arrived at up to 20 times lower values of $E^{*}$ (Hora et al., 2008).

After initial results with one-fluid hydrodynamics (Hora, 2009) were performed it was important to receive more information from applying the genuine two-fluid hydrodynamics

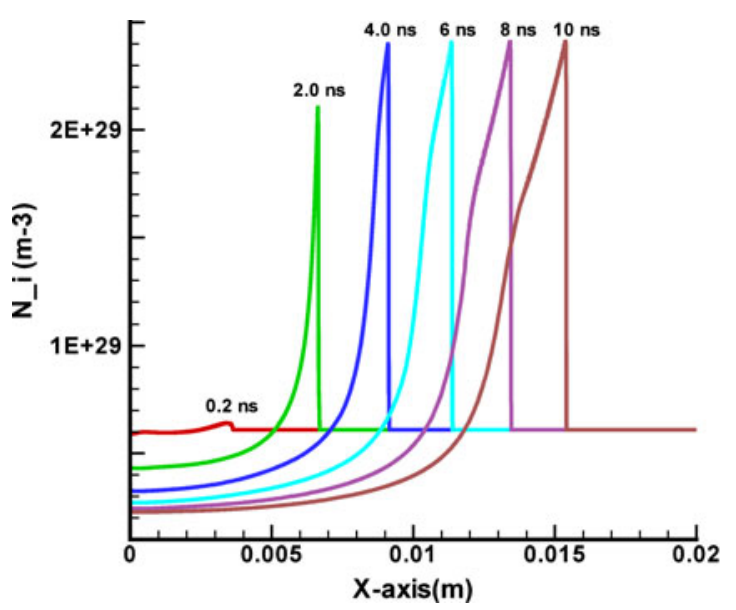

Fig. 5. Ion densities $N_{\mathrm{i}}$ depending of the depth $X$ after a $1 \mathrm{ps} 3 \times 10^{8} \mathrm{~J} / \mathrm{cm}^{2}$ laser pulse of $248 \mathrm{~nm}$ wavelength has irradiated solid density DT. Parameter is the time after the ps interaction. 


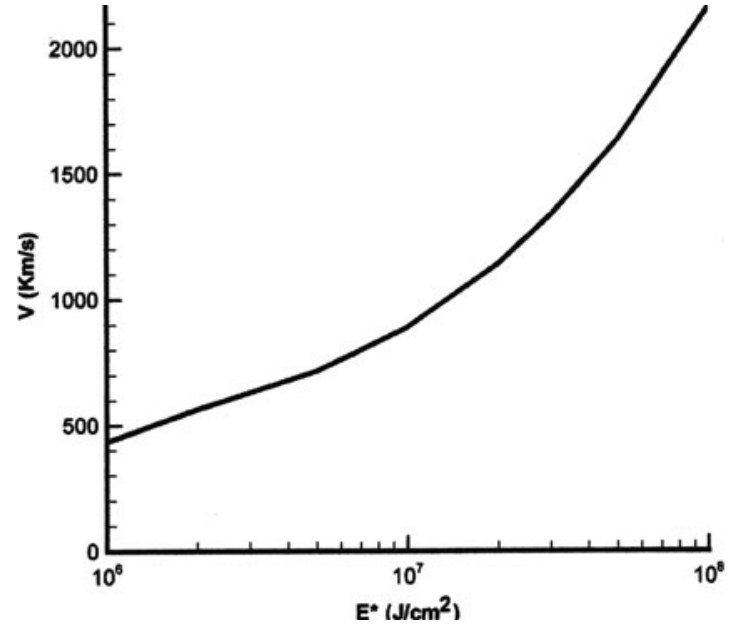

Fig. 6. Velocity $V$ of the fusion flame at $2 \mathrm{~ns}$ after the initiating ps laser pulse of $248 \mathrm{~nm}$ wavelength depending on the energy flux $E^{*}$.

(Lalousis \& Hora, 1983; Hora et al., 1984) about the dynamical generation of internal electric fields and of shock processes (Betti et al., 2007) and diffusion. Using the same parameters for the generation of ps ultrahigh-accelerated plasma blocks of about a $5 \mu \mathrm{m}$ depth moving into solid density DT, we are summarizing here further reported results following the initial computations (Lalousis \& Hora, 1983; Hora et al., 1984). Figure 5 shows ion densities with shock profiles moving at the interaction edge into the solid density DT. A shock structure shows maximum densities four time the solid state as it is the result of the Rankine-Hugoniot simplified analytical model. The shock thickness is increasing on time what could be covered only by complete inclusion of thermal and plasma properties in our genuine two-fluid computation (Lalousis \& Hora, 1983; Hora et al., 1984). It is interesting that the shock is pronounced only very late after $2 \mathrm{~ns}$ being only little visible at

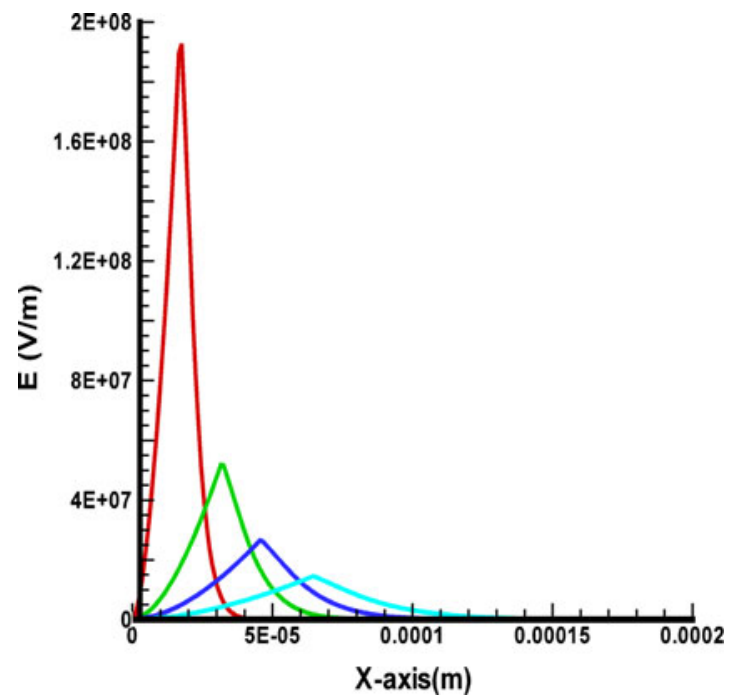

Fig. 7. Longitudinal electric field $E$ as in case of Figure 1 for $E^{*}=10^{8} \mathrm{~J} /$ $\mathrm{cm}^{2}$ depending of the depth $X$ at times of $40 \mathrm{ps} ; 400 \mathrm{ps} ; 1 \mathrm{~ns} ; 2 \mathrm{~ns}$ from the highest maximum, respectively.
200 ps. It should be mentioned that the same plots for three times lower $E^{*}$ values shows an about two times earlier building up of the shock. The shock velocity was evaluated for varying $E^{*}$ and resulted in Figure 6 at $2 \mathrm{~ns}$. It can be seen that the fast increasing velocity at $E^{*}$ of $10^{8} \mathrm{~J} / \mathrm{cm}^{2}$ is reaching values higher than $2000 \mathrm{~km} / \mathrm{s}$ with convincing ignition properties. In order to understand the reasons for the long ( $>200 \mathrm{ps}$ ) delay of the shocking process, we show in Figure 7 the longitudinal electric field which can be seen only form the genuine two-fluid hydrodynamics. It is remarkable that during the very first few hundred ps after the laser pulse in the phase of establishing the shock front, the electric field is rather high and decays only at 2 ps to comparably low values when the shock is nearly fully developed (Fig. 5).

The studies with the genuine two-fluid hydrodynamics permits an evaluation about the decreasing velocity of the front of the fusion flame at the very long times of ns after the ps initiation of the flame. Figure 8 shows the results of fusion reaction rates with a 1 ps laser pulse of $E^{*}=10^{8} \mathrm{~J} /$ $\mathrm{cm}^{2}$. The velocity of the fusion flame is decreasing from $2130 \mathrm{~km} / \mathrm{s}$ at $2 \mathrm{~ns}$ to $1070 \mathrm{~km} / \mathrm{s}$ at $10 \mathrm{~ns}$ and the values of the reaction rates decay faster than in the case with only three times higher irradiation. Nevertheless, the fusion gains are not very much lower, going down at $10 \mathrm{~ns}$. The gains at the time of $2 \mathrm{~ns}$ are nearly the same. The result of the ion density at the flame front has reached nearly the Rankine-Hugoniot value of four times compression earlier at $2 \mathrm{~ns}$ than in the case with three times higher irradiation of Figure 5.

The fact of the growing thickness of the compression range on time may be understood by the thermal mechanisms around the fusion flame with a stronger effect at the first case with the higher energy flux. This can also be understood that the shock compression in the early stage appears within a too thin area such that the thermal mechanisms - completely

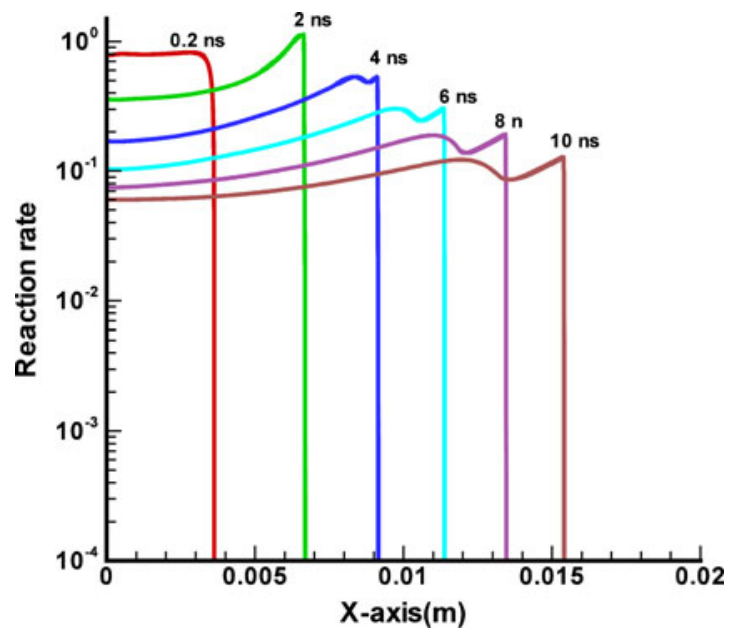

Fig. 8. Reaction rates (to be multiplied by $10^{36} \mathrm{~m}^{-3} / \mathrm{s}$ ) in solid DT at $1 \mathrm{ps}$ pulse of energy flux $E^{*}=3 \times 10^{8} \mathrm{~J} / \mathrm{cm}^{2} \mathrm{KrF}$ laser irradiation depending on the fuel depth $\times$ at different times up to $10 \mathrm{~ns}$. 
covered by the code - are causing a dissipation of the too thin compression areas.

We may conclude that the earlier studied mechanisms of shock ignition (Betti et al., 2007) for fusion as evaluated for longer ns pulses is supported by our results at least for these longer times (Eliezer et al., 2014). This supports the results of shock wave ignition for fusion (Betti et al., 2007) which requires a long (few ns) and high-energy laser pulse. The high pressure $(P)$ shock wave thickness $(d)$ in the ns interaction case is estimated from the equality of pressures at the interface between a flyer (or a plasma block with density $\rho_{0}$ and flow velocity $u_{0}$ ) and the compressed target (the nuclear fuel: DT or $\mathrm{pB} 11$, etc. with density $\rho_{\mathrm{C}}$ and flow velocity $\left.u_{\mathrm{C}}\right)$. Since $P \sim \rho_{0} u_{0}^{2} \sim \rho_{\mathrm{C}} u_{\mathrm{C}}^{2}$ and the shock wave transition time in the flyer with a thickness $l$ is $t=l / u_{0}$, one gets a shock wave thickness $d$ of the order of $d \sim\left(\rho_{0} / \rho_{\mathrm{C}}\right)^{1 / 2} l$. In this purely shock ignition scheme - in contrast to the here studied ps plasma block ignition - the ns case values are $\left(\rho_{0} / \rho_{\mathrm{C}}\right) \sim 0.001$ and $l \sim 1 \mu \mathrm{m}$ implying $d \sim 0.03 \mu \mathrm{m}$. The ignition criterion is based on the requirement that the $\alpha$-particles created in the DT reaction are reabsorbed in the hot spot implying a " $\rho R$ " value larger than $0.3 \mathrm{~g} / \mathrm{cm}^{2}$ for a temperature about $10 \mathrm{keV}$ and larger values for higher temperatures. In the shock wave ignition, " $\rho R$ " $=\rho_{\mathrm{C}} d \sim$ $l\left(\rho_{0} \rho_{\mathrm{C}}\right)^{1 / 2} \sim 0.003 \mathrm{~g} / \mathrm{cm}^{2}$ for our case which is two order of magnitude too low. This result fully explains why the ns interaction scheme for shock ignition (Lalousis \& Hora, 1983; Hora et al., 1984; Betti et al., 2007) is basically different from the here discussed ps laser pulse-initiated blockignition scheme.

It was very surprising when extending these very detailed and established results for DT fusion were applied to the fusion of HB11. Against all knowledge that fusion based on binary reactions as used for DT, the threshold $E^{*}$ arrived at about the same values for HB11. It was most unexpected and curious, that this case had a threshold $E^{*}$ of a similar level as DT (Hora et al., 2009; 2010). In addition, it should be underlined that the calculations for HB11 were pessimistically performed in the same way with binary nuclear reactions only as in the case of DT because the secondary reactions of the avalanche process (see the second section of this paper) were not known at this time.

Though these calculations were based only on plane wave geometry (Hora et al., 2009; 2010), the clarification of the basic change of the earlier known extreme difficulties of HB11 fusion against DT resulting now for the block ignition for HB11 as "no difficulties" was interpreted as: "This has the potential to be the best route to fusion energy" (Haan, 2010).

Summarizing the block ignition, the ps ultrahigh-power laser ignition of fusion with the non-thermal direct conversion of the laser pulse energy into mechanical motion of plasma blocks is essentially different from the fusion with $\mathrm{ns}$ pulse ignition of fusion based on thermal interaction. In the ns case, problems are with thermalizing collision processes, delaying equipartition for ion heating, instabilities, etc. This difference is simply seen form Eq. (5), that the ns process is based on the first term of the force density and the ps process is based on the second term of non-thermal ps interaction with directly converting of the optical laser energy into macroscopic mechanical motion of the plasma blocks. This fundamental difference is including the principle of non-linearity (see reference to Feynman, 1987) in relation to the prediction of the block generation by the non-linear force (Fig. 3) and measured first by Sauerbrey (1996) as ultrahigh acceleration of the plasma blocks (Hora et al., 2007). The accelerations were more than 10,000 times higher than measured before in laboratories. This could then be used for the side-on ignition of Chu (1972) and Bobin (1974) ps fusion-flame initiation; however, only after the CPA of shorter than ps laser pulses was available (Strickland \& Mourou, 1985; Mourou et al., 1998).

It should be underlined explicitly that the here used ps process of block ignition follows a different principle of interaction. Instead of the heating with ns pulses, the non-thermal electrodynamic non-linear force interaction is a macroscopic process known from the specific microscopic quantum properties, reducing instabilities, delays for thermal equipartion processes, and chaotic thermostatistics. This differentiation was also illustrated by Edward Teller und Lord May of Oxford; see citations in the paper of Hora (2011).

\section{AVALANCHE BLOCK IGNITION WITH ULTRAHIGH MAGNETIC FIELDS}

The just reported application of experimentally confirmed ultrahigh acceleration of plasma blocks to be used for computation of block ignition of fusion, by non-linear force-driven ps laser pulses, was based on plane geometry interaction. For thin laser beams, the energy losses in the solid targets have to be taken into account. The first steps were to study spherical generation of ps-block ignition for DTand HB11 fusion, where, however, the reached gains were not sufficiently high and a cylindrical geometry with magnetic field trapping was the next step.

Documented before the preparations to the 2012 IAEA Fusion Energy Conference (Hora et al., 2012a), the spherical irradiation has been studied with shock generation and increase of density (Hora et al., 2014a) with similar results to Malekynia and Razipur (2013). For generating spherical converging ps laser pulses of more than EW (exawatt $=$ $10^{18} \mathrm{~W}$ ) power, this can be combined with the advantages which were opened using fiber glass generation of CPA (Mourou et al., 1998; Moustaizis et al., 2013; Hora et al., 2014a) amplified laser pulses as developed by the ICAN Project (Mourou et al., 2013). This is also of interest for other uses than for fusion energy generation as electron or hadron accelerators with many applications including cancer therapy (Banati et al., 2014), as well as for generating $\mathrm{PeV}$ particles or for conditions of high-energy physics for exploring extremely high field processes as pair production in vacuum. This is also of interest for a fusion power station to overcome problems of lateral energy losses of the plane 


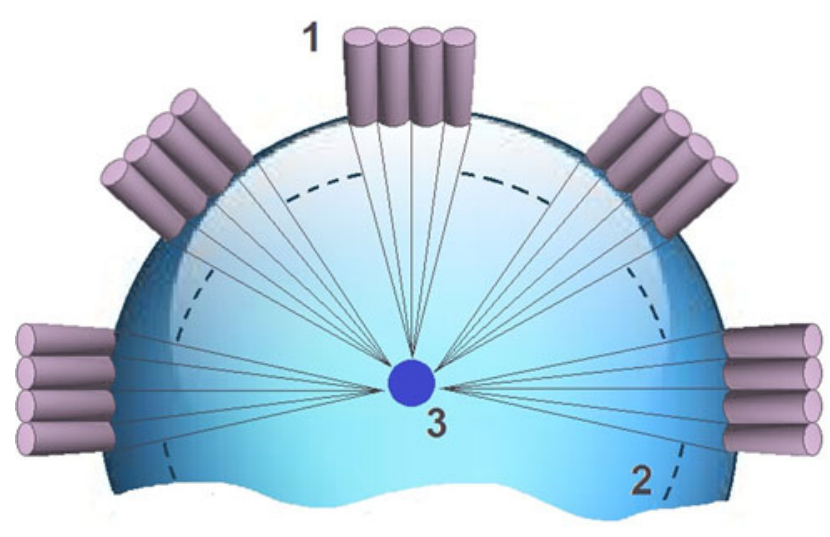

Fig. 9. Generation of a spherical shrinking laser pulse from radially directed fiber ends at a radius 1 to irradiate a spherical solid state fusion fuel 3 with the concentric grid 2 of positive electric charge for slowing down the generated a-particles from the HB11 reactions (Hora et al., 2014a).

wave front geometry of the ICAN fiber laser (Mourou et al., 2013 ) to be developed into spherical geometry (Hora et al., $2014 a$ ) as can be seen from Figure 9.

The output of the fibers at a sphere 1 of Figure 9 of the amplified beam of the fibers can be directed radially without needing any further optics. The wave front is of such a highquality single-mode focusing which has (Mourou et al., 2013) to go to a diameter of $10 \mu \mathrm{m}$ such that intensities at about $10^{21} \mathrm{~W} / \mathrm{cm}^{2}$ are reached when the spherically converging laser pulse the surface of a target 3 . The fibers automatically permit an exceptionally high quality of single-mode beam uniformity where the usually undesired maxima in the beam profile with glass lasers are automatic eliminated due to the fiber optics quality. The resulting gains for irradiation of uncompressed solid density fuel of DT or of HB11 including avalanche reactions with subsequent dynamic compression was evaluated needing ps laser pulses of EW for fusion gains of few hundred where limitations are given by the limited spherical fuel geometry (Hora

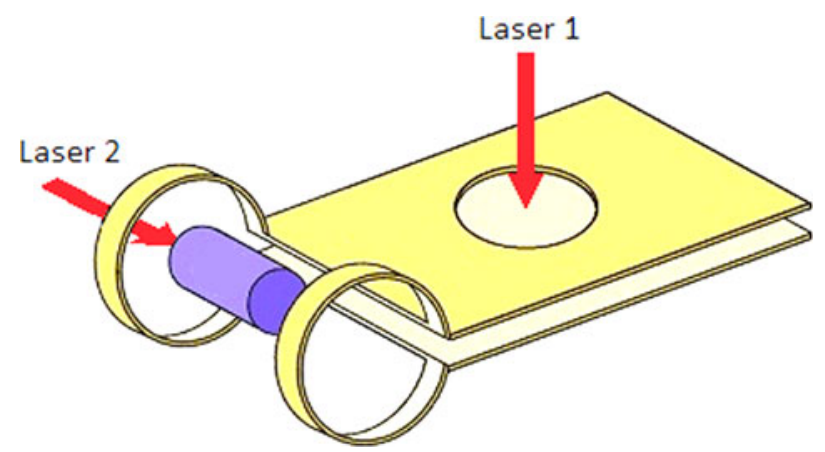

Fig. 10. Generation of a $5 \mathrm{kT}$ magnetic field in the coils (Fujioka et al., 2013) by firing a $>\mathrm{kJ}$ ns laser pulse 1 into the hole between the plates. The HB11 fusion fuel is coaxially located in the coils and the block ignition of the fusion flame is produced by the ps laser pulse for block ignition from laser 2. et al., 2014a). This case is not of interest for the following consideration.

Another option instead of spherical geometry is to consider cylindrical geometry for the reaction by application of an external pulsed ultrahigh magnetic field. This can be produced by the interaction between of an ns laser beam with a capacitor coil target (Fig. 10). This mechanism has been described and experimentally demonstrated for the first time in 1984 (Daido et al., 1986) and recently improved by Fujioka et al. (2013) at Gekko XII facility. More details about measurements of the ultrahigh magnetic fields were reported by Santos et al. (2015). These magnetic fields with intensities of few kilotesla (kT), contained in few cubic millimeters and with time duration of few ns, generated with laser pulses above $\mathrm{kJ}$, can be employed to trap the cylindrical plasma generated in the HB11 target for a fusion reaction in the coil of Figure 10.

The computation without involving secondary avalanche reactions - only with binary HB11 reactions - shows sufficient trapping by a cylindrical $10 \mathrm{kT}$ magnetic field from fluid hydrodynamics (Lalousis et al., 2014; 2015).

The following two-fluid computations use a solid cylinder of $1 \mathrm{~cm}$ in length and $1 \mathrm{~mm}$ radius as a coaxial HB11 fuel target in the coil of Figure 10 where the generated $\alpha$-particles are included as a third fluid using a magnetic field of $10 \mathrm{kT}$. The mean gyroradius of the a-particles from the HB11 reaction in a $10 \mathrm{kT}$ field is about $43 \mu \mathrm{m}$. This is sufficiently small compared with the $1 \mathrm{~mm}$ radius of the cylinder to achieve strong magnetic field confinement of the alphas. The radial dependence at the time of $100 \mathrm{ps}$ is shown in Figures 11-13 and the propagation of the fusion along the cylindrical axis results in a length $x \sim 0.83 \mathrm{~cm}$ within $1 \mathrm{~ns}$ (Fig. 14). Owing to the low electron temperatures (Fig. 12) the cyclotron radiation generated in the cylindrical reaction volume

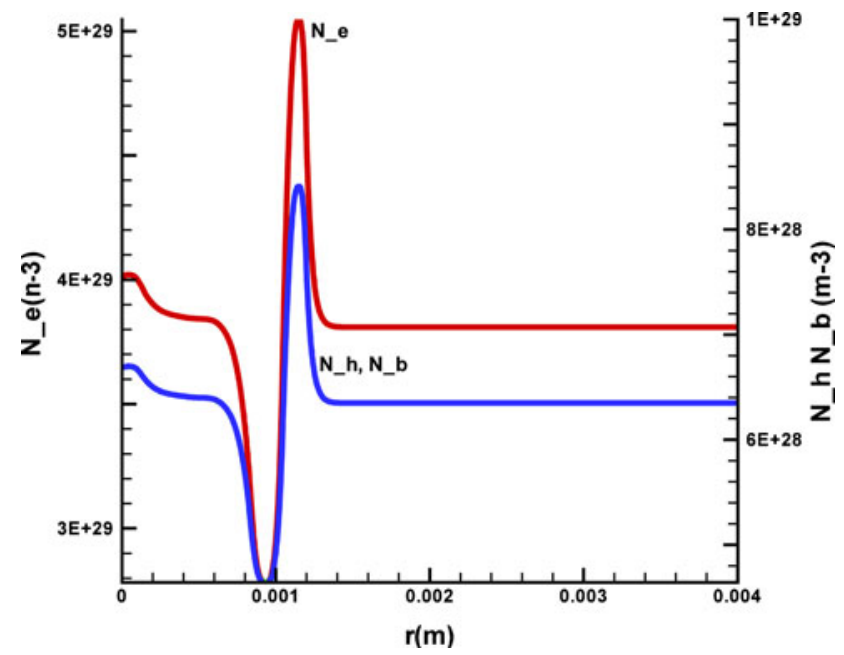

Fig. 11. Solid $\mathrm{HB} 11$ cylinder of $1 \mathrm{~mm}$ radius irradiated by a $\mathrm{ps}-10^{20} \mathrm{~W} / \mathrm{cm}^{2}$ laser pulse of $248 \mathrm{~nm}$ wavelength at concentric radius of $1 \mathrm{~mm}$. After $100 \mathrm{ps,}$ the dependence on the radius $r$ is shown for the density of electrons $N_{\mathrm{e}}$, boron $N_{\mathrm{b}}$ and hydrogen $N_{\mathrm{h}}$ (sequence at $r=0$ from above) using a $10 \mathrm{kT}$ magnetic field (Lalousis et al., 2015). 


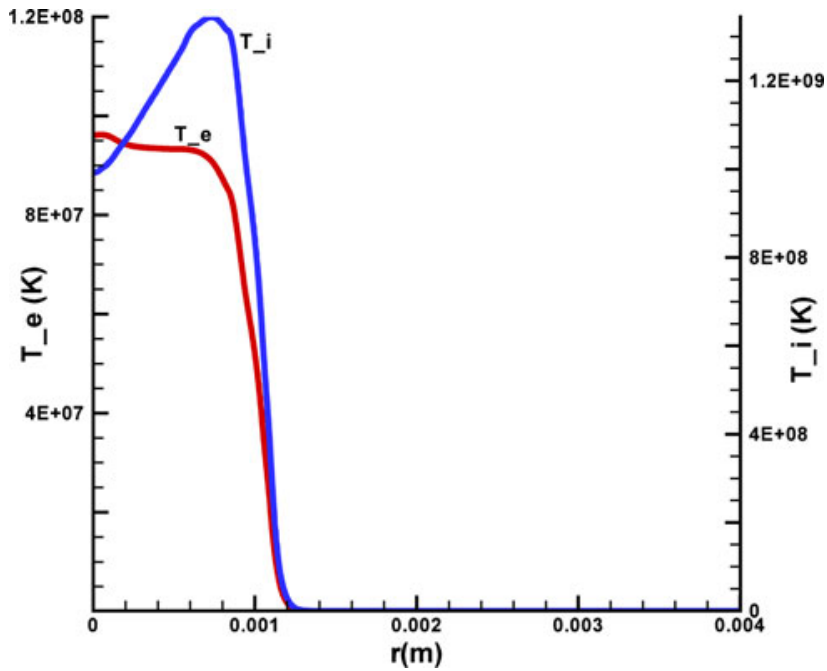

Fig. 12. Same as Figure 11 with electron and ion temperatures.

(Lalousis et al., 2015) is $<3 \mathrm{~kJ} / \mathrm{ns}$ (Gulkis, 1987). Thus cyclotron radiation losses can be neglected.

Figure 13 shows the radial dependence of the $\alpha$-particle density and of the magnetic field parallel to the cylindrical axis at the time of $100 \mathrm{ps}$. While the alphas are well confined to the initial radius of $1 \mathrm{~mm}$ and the magnetic field is modified at this periphery only due to $E \times B$ rotation, etc. For the propagation of the fusion flame along the axis of the cylinder, the one-dimensional (1D) computation of the reaction rate is shown in Figure 14. The total number of $\alpha$-particles depending on the square of $r$ has been increased considerably showing the successful ignition though only binary reactions were included in the computations. If one included the preliminary approximation of avalanche reactions the reaction

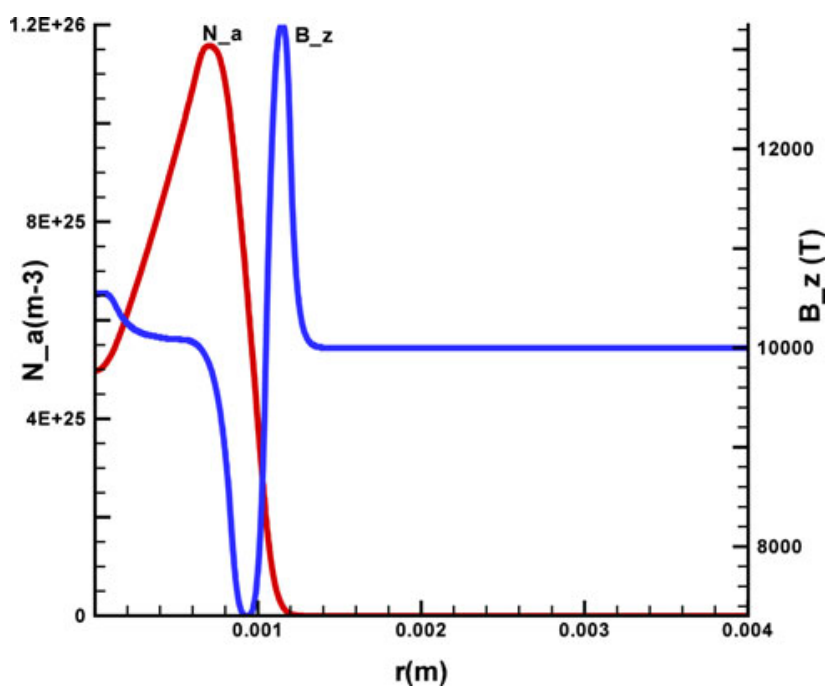

Fig. 13. Same case as Figure 11: Solid HB11 cylinder of $1 \mathrm{~mm}$ radius irradiated by $\mathrm{ps}-10^{20} \mathrm{~W} / \mathrm{cm}^{2}$ laser pulse of $248 \mathrm{~nm}$ wavelength. Radial density at $100 \mathrm{ps}$ of primary produced $\alpha$-particles $\mathrm{N} \_$a ending at $1 \mathrm{~mm}$ laser irradiation radius and the magnetic field strength depending on the radius $r$ (in untouched fuel $10 \mathrm{kT}$ at radius above $1 \mathrm{~mm}$ ).

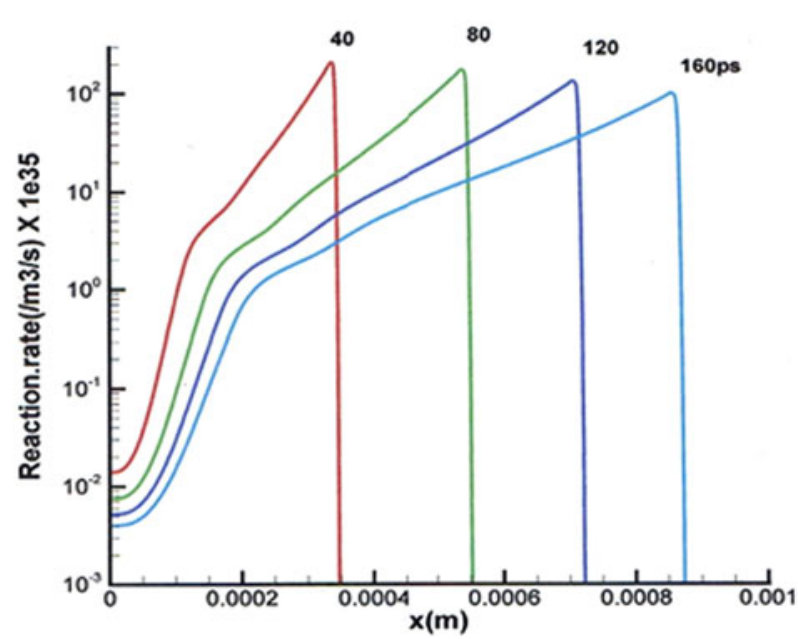

Fig. 14. HB11 fusion reaction rate at different depths $x$ in 1D computation parallel to the magnetic field, at times after the ps generation of the fusion flame initiated by $10^{20} \mathrm{~W} / \mathrm{cm}^{2}$, ps, $248 \mathrm{~nm}$ wavelength laser pulse.

result is rather modest taking into account that a $3 \mathrm{EW}-\mathrm{ps}$ laser was needed to reach a reaction gain in the range above 500 .

If one could assume the additional avalanche process, a solid fuel cylinder of $1 \mathrm{~cm}$ length and $2 \mathrm{~mm}$ diameter blockignited by a $3 \mathrm{MJ}$-ps laser pulse of $3 \mathrm{EW}$ power will produce more than 1 gigawatt (GW) electrical energy (Lalousis et al., 2014) again with a gain only of about 300 . The produced a-particles are gyrating with a radius of $43 \mu \mathrm{m}$ which type of particle motion is not included in the hydrodynamics though most of the relevant plasma mechanisms were sufficient to such extend that the essential dynamic and confining processes are covered.

The same computations with a B11 fuel cylinder of $1 \mathrm{~cm}$ length and $0.2 \mathrm{~mm}$ diameter (Lalousis et al., 2014) resulted in some radial expansion of the plasma with binary reactions

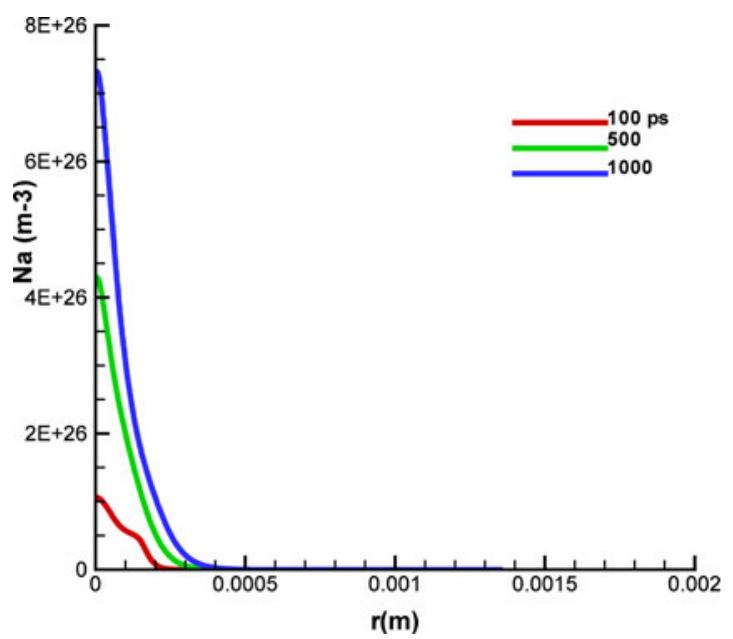

Fig. 15. a density $N_{\mathrm{a}}$ depending on the radius $r$ at different times (from lowest to highest curves for 100,500 and $1000 \mathrm{ps}$, respectively) showing ignition from the increase of the curves on time (Lalousis et al., 2014). 
as shown in Figure 15 with the radial distribution of the a-particles during times up to $1000 \mathrm{ps}$. Though this was only for binary reactions, the ignition process is evident. For the case that avalanche reactions are included for irradiation with $10^{20} \mathrm{~W} / \mathrm{cm}^{2}$ at the axis of $0.2 \mathrm{~mm}$ diameter on the HB11 solid fuel cylinder of $1 \mathrm{~cm}$ length and $1 \mathrm{~mm}$ radius, it is estimated that the laser pulse 2 (Fig. 10) of $30 \mathrm{~kJ}$ energy and $1 \mathrm{ps}$ duration (30 PW power) is producing more than 1 GJ energy. The estimation is based on excluding the a gyration for the hydrodynamics. Though the genuine many-fluid codes takes care of highly non-equilibrium plasma conditions (Fortov \& Lakubov, 1998; Davidson, 2001), it may be preferred to base further steps on specific experiments of HB11 reactions following the preceding measurements (Korn et al., 2014; Picciotto et al., 2014; Margarone et al., 2015).

\section{POSSIBLE ECONOMIC CLEAN AND SAFE FUSION REACTOR}

The experimental proof of the avalanche reactions for HB11 (Hora et al., 2014a; Korn et al., 2014) results in the scheme for combination of the non-linear force-driven plasma block ignition with ultrahigh accelerations together with the ultrahigh magnetic fields for cylindrical trapping of the fusion reaction. In the case for igniting $1 \mathrm{~cm}$ long cylinder of HB11 of $1 \mathrm{~mm}$ radius can then be based on of the irradiation by the

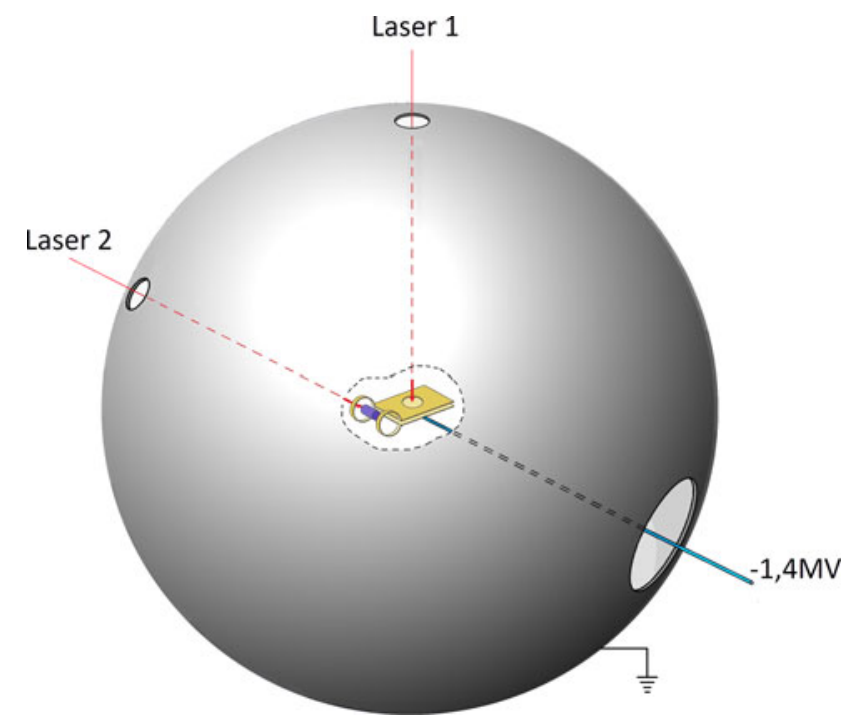

Fig. 16. The HB11 fusion without a radioactive radiation problem is based on a block ignition Hora et al. (2007) with a $30 \mathrm{~kJ}$-ps laser pulse 2 (Fig. 10) where the solid hydrogen-boron fuel in the cylindrical axis of the magnetic coil is trapped by a $10 \mathrm{kT}$ field sustained for about $1 \mathrm{~ns}$ after being generated by a laser pulse 1 of comparable duration. The central reaction unit (Fig. 10) is electric charged to the level of -1.4 million volts against the wall of a sphere producing $\alpha$-particles (He nuclei) of more than a GJ energy, of which a small part is needed for the operation of the laser pulses. One part of the gained costs of electricity is needed for the apparatus of the central reaction and for the boron metal of the fuel being destroyed at each reaction (Hora et al., 2014a). laser pulse 2, Figure 10, with the laser beam diameter of $200 \mu \mathrm{m}$ and an intensity of $10^{20} \mathrm{~W} / \mathrm{cm}^{2}$ of a $30 \mathrm{PW}$ pulse of ps duration. The computation of the $\alpha$-particle generation - even very pessimistically using only binary reactions as in the DT case - as shown in Figure 16 with a $10 \mathrm{kT}$ magnetic field results in the progress of the reaction gain.

There is a comparably slow expansion of the growing a-particle density generated by the fusion reaction against the magnetic field. Including the avalanche reactions into the $1 \mathrm{~ns}$ reaction results in the production of considerably higher gain of 1 Gigajoule energy in the generated of initially mono-energetic generated $\alpha$-particles generated by the laser pulse 2 (Fig. 10) needing only $30 \mathrm{~kJ}$ energy (Hora et al., $2014 b$ ). On the basis of an avalanche process during up to $1 \mathrm{~ns}$ duration, the energy in the a-particles is more than $1 \mathrm{GJ}(277 \mathrm{kWh})$. For conversion of most of the $2.99 \mathrm{MeV}$ a-particle energy into electrostatic energy, the particles have to be slowed down by an electric field of $-1.4 \mathrm{MV}$. This field has to be generated at each reaction between the central reaction unit against the spherical mantle of the reactor of Figure 16.

For a power station the main part of the generated energy of the a-particles from the level of a $-1.4 \mathrm{MV}$ voltage into polyphase alternating current can be performed as known from the megavolt-direct-current transmission line techniques Kanngiesser (1994). If the reactor works with a frequency of $1 \mathrm{~Hz}$, the electric current for conversion is 714 Amp averaged between each fusion reaction.

\section{SUMMARY}

Combining the experimental result of most exceptionally high gains of proton-boron (HB11) fusion (Korn et al., 2014; Picciotto et al., 2014; Margarone et al., 2015) based on the concluded avalanche mechanisms (Hora et al, $2014 b$; 2015) and evaluated now in more details, with the results of $>$ PW-ps laser-driven block ignition (Hora et al., 2010; Lalousis et al., 2014) was leading to a first step of producing ultraclean GJ fusion energy possibly by $30 \mathrm{~kJ}$ laser irradiation. Cylindrical magnetic trapping with ultrahigh magnetic fields in the $10 \mathrm{kT}$ range is necessary. The open treatment of these developments was approved (Hora, 2014) to follow the route for designing of an economic longlasting energy source. This furthermore has to be based on appropriate scientific and technological explorations.

The presented results are the work of more than 40 years research (Hora, 1969; 2000; 2009; Hora et al., 2014b; 2015) possible only after providing extremely high-power ps laser pulses (Strickland \& Mourou, 1985; Mourou et al., 1998) for demonstration of the predicted picoseocond block acceleration (Sauerbrey, 1996) and ignition with ultrahigh magnetic fields (Fujioka et al., 2013). The key result is the here detailed evaluation of the highly increased reaction rate for HB11 fusion (Korn et al., 2014) as an avalanche process (Hora et al., 2014b; 2015). This is not the end but may be the beginning of large-scale research to one of possible options for 
environmentally acceptable ultraclean and economic energy production.

\section{REFERENCES}

Banati, R. Hora, H., Lalousis, P. \& Moustaizis, S. (2014). Ultrahigh laser acceleration of plasma blocks with ultrahigh ion density for fusion and hadron therapy. J. Intense Pulsed Laser Appl. Adv. Phys. 4, 11-16.

Belyaev, V.S., Matafonov, A.P., Vinogradov, V.I., Krainov, V.P., Lisitsa, V.S., Roussetski, A.S., Ignatyev, G.N. \& Andrianov, V.P. (2005). Observation of neutronless fusion reactions in picosecond laser plasmas. Phys. Rev. E 72, 026406.

Betti, R., Chou, C.D., Anderson, K.S., Perkins, L.J., Theobald, W. $\&$ Sodolov, A.A. (2007). Shock ignition of thermonuclear fuel with high areal density. Phys. Rev. Lett. 98, 155001.

BobIN, J.-L. (1974). Nuclear fusion reactions in fronts propagating in solid DT. (Schwarz, H. and Hora, H., Eds.) Laser Interaction and Related Plasma Phenomena, Vol. 4B, p. 465. New York: Plenum Press.

Boreham, B.W., Newman, D.S., HöPfl, R. \& Hora, H. (1995). Depressed photoemission from Görlich cathodes at high laser light intensities. J. Appl. Phys. 78, 5848-5850.

ButLER, D. (1996). Now Europe's physicists seek shift in strategic fusion research. Nature 380, 655.

CAmpbell, E.M. (2006). High intensity laser plasma interaction and application to inertial fusion and high energy density physics D.Sc. (Doctor of Science) Thesis. Sydney, Australia: University of Western Sydney, 723 p.

CHu, M.S. (1972). Thermonuclear reactions waves at high densities. Phys. Fluids 15, 412.

Cicchitelli, L., Hora, H. \& Postle, R. (1990). Longitudinal field components of laser beams in vacuum. Phys. Rev. A 41, 3727-3732.

Daido, H., Miki, F., Mima, K., FujtTa, M., Sawaki, K., Fujita, H., Kitagawa, Y., NaKai, S. \& YamanaKa, C. (1986). Generation of a strong magnetic field by an intense $\mathrm{CO}_{2}$-laser pulse. Phys. Rev. Lett. 56, 846.

DAvidson, R. (2001). Nonneutral Plasmas. Singapore: World Scientific.

Eliezer, S., Nissim, N., Martinez Val, J.M., Mima, K. \& Hora, H. (2014). Double layer acceleration by laser radiation. Laser Part. Beams 32, 211-217.

Feynman, R. (1987). see H. Hora, Laser Plasma Physics, SPIE Books, Belllingham WA 2000, Section 6.3; Hora. H. Laser and Particle Beams 29, 275 (2011).

Földes, I.B., Bakos, J.S., Gal, K., Juhasz, Y., Kedves, M.A., Koscis, G., Szatmari, S. \& Veres, G. (2000). Properties of high Harmonics generation by UV laser pulses on solid surfaces. Laser Phys. 10, 264.

Fortov, V.E. \& LaKubov (1998). Physics of Nonideal Plasmas. Singapore: World Scientific.

Fujioka, S., Zhang, Z. Ishihara, K., Shigemori, K., Hironaka, Y., Johzaki, T., Sunahara, A., Yamamoto, N., NaKashima, H., Watanabe, T., Shiraga, H., Nishimura, H. \& Azechi, H. (2013). Kilotesla magnetic field due to a capacitor-coil target driven by high power laser. Sci. Rep. 3, 1170-1176.

Gulkis, S. (1987). In Encyclopaedia of Physical Science and Technology (Meyers, R.A., Ed.), Vol. 11, p. 644. San Diego: Academic Press, Eq. (33).
HaAn, S. (2010). see in Li Yuandi, Editor, Nuclear Power without radioactivity Highlights in Chemical Technology, 24 March 2010 Royal Soc. Chemistry, London; or A. Calilasseria. Physics. Rational Skepticism Forum, see google "boron fusion", (22 April 2010) http://www.physorg.com/news190295239.html.

HoHLA, K. \& Kompa, K.L. (1973). Gigawatt photochemical iodine laser. Appl. Phys. Lett. 22, 77-80.

Hora, H. (1969). Nonlinear confining and deconfining forces associated with the interaction of laser radiation with plasma. Phys. Fluids 12, 182-191.

Hora, H. (1981). Physics of Laser Driven Plasma. New York: Wiley, figures $10.18 \mathrm{a} \& \mathrm{~b}$.

HorA, H. (1985). The transient electrodynamic forces at laser-plasma Interaction. Phys. Fluids 28, 3706-3707.

Hora, H. (2000). Laser Plasma Physics. Belllingham, WA: SPIE Books.

HorA, H. (2003). Skin-depth theory explaining anomalous picosecond-terawatt laser plasma interaction II. Czech. J. Phys. 53, 199-217.

HorA, H. (2009). Laser fusion with nonlinear force driven plasma blocks: thresholds and dielectric effects. Laser Part. Beams 29, 207-222.

Hora, H. (2011). Distingished celebration for Professor George H. Miley by the University of Illinois, Urbana Illinois, USA. Laser Part. Beams 29, 275-278.

Hora, H. (2013a). Extraodrinary jump of increasing laser fusion gains experienced at volume ignition for combination with NIF experiments. Laser Part. Beams 31, 229-232.

Hora, H. (2013b). Neutron-free production of nuclear fusion reactions without additional fields. German Patent Application 10 2013016306.6

Hora, H. (2014). Permission for open use of German Patent application 102013016 305.6. 15 Jan.

Hora, H., BadziaK, J., Boody, F.P., HöPfl, R., JungwiRth, K., KRAlikowa, B., Kraska, J., Laska, L., Parys, P., Perina, V., Pfeifer, M., Rohlena, K., Skala, J., Ullschmied, J., Wolowski, J. \& Woryna, E. (2002a). Effects of ps and ns. laser pulses for giant ion source. Opt. Commun. 207, 333-337.

Hora, H., Badziak, J., Read, M.N., Li, Y.-T., Liang, T.-J., Liu, H., Sheng, Z.-M., Zhang, J., Osman, F., Miley, G.H., Zhang, W., He, X., Peng, H., Osman, F., Glowacz, S., Jablonski, S., WolowSki, S., Skladanowski, Z., Jungwirth, K., Rohlena, K. \& Ullschmied, J. (2007). Fast ignition by laser driven beams of very high intensity. Phys. Plasmas 14, 072701/1-7.

Hora, H., Castillo, R., Clark, R.G., Kane, E.L., Lawrence, V.E., Miller, R.D.C., Nicholson-Florence, M.F., NovaK, M.M., Ray, P.S., SHEPANSKI, J.R. \& TsIVINSKY, I. (1978). Calculation of intertal confienement fusion gains using a collective model for reahat, bremsstrahlung and fuel depletion for higly efficient elecgtrodynamic laser compressions. Plasma Phys. Control. Nucl. Fusion Res. Innsbruck 1978 Nucl. Fusion (Suppl. 1979) 3, 237-246.

Hora, H. \& Kelly, J.C. (2009). Nuclear energy without radioactivity. Austr. Phys. 46, 111-113.

Hora, H., Lalousis, P. \& Eliezer, S. (1984). Analysis of the inverted double layers in nonlinear force produced cavitons at laserplasma interaction. Phys. Rev. Lett. 53, 1650-1652.

Hora, H., Lalousis, P., Eliezer, S., Miley, G.H., Moustaizis, S. \& Mourou, G. (2014b). New option for solving the climatic problems with non-thermal laser driven boron fusion and ultrahigh magnetic fields. arXiv 1412.4190, see also N. Byrne, E. Hunt 
and M. Beilharz (2014). News Release Austr. Physics Congress December 2014, niall@sciencepublic.com.au.

Hora, H., Lalousis, P., Guiffrida, L., Margarone, D., Korn, G., Eliezer, S., Miler, G.H., Moustaizis, S. \& Mourou, G. (2015) Petawatt laser pulses for proton-boron high gain fusion with avalanche reactions excluding problems of nuclear radiation. SPIE Proceedings 9515, 951518/1-15.

Hora, H., Lalousis, P. \& Moustaizis, S. (2014a). Fiber ICAN laser with exawatt-picosecond pulses for fusion without nuclear radiation problems. 32, 63-68.

Hora, H., Lalousis, P., Moustaizis, S., Földes, I., Miley, G.H., Yang, X., He, X.T., Eliezer, S. \& Martinez-Val, J.-M. (2012a). Shock Studies in Nonlinear Force Driven Laser Fusion with Ultrahigh Plasma Block Acceleration. IAEA Proc. Fusion Energy, San Diego October 2012. Paper IFE/P6-03, 8 pages (IAEA Vienna 2013) http://www-naweb.iaea.org/napc/ physics/FEC/FEC2012/papers/27_IFEP603.pdf.

Hora, H., Malekynia, B., Ghoranneviss, M., Miley, G.H. \& He, X. (2008). Twenty times lower ignition thresholds for laser driven fusion using collective effects and the inhibition factor. Appl. Phys. Lett. 93, 011101/1-3.

Hora, H., Miley, G.H., Ghoranneviss, M., Malekynia, B. \& Azizi, N. (2009) Laser-optical path to nuclear energy without radioactivity: fusion of hydrogen-boron by nonlinear force driven plasma blocks. Opt. Commun. 283, 4124-4126.

Hora, H., Miley, G.H., Ghorannviss, M., Malekynia, H., Azizi, N. \& He, X-T. (2010). Fusion energy without radioactivity: laser ignition of solid hydrogen-boron(11) fuel. Energy Environ. Sci. 3, 479-486.

Hora, H., Peng, H.-S., Zhang, W. \& Osman, F. (2002b). New skin depth interaction by ps-TW laser pulses and consequences for fusion energy. SPIE Proc. 4914, 42-48.

Hora, H., Sadighi-Bonabi, R., Yazdani, R., Afaideh, A., Nafari, F. \& GHoranneviss, M. (2012b). Effect of quantum correction of the acceleration and delayed heating of plasma blocks. Phys. Rev. E 85, 036404/1-6.

Hurricane, O.A., Callahan, D.A., Casey, D.T., Celliers, P.M., Cerjan, C., Dewald, E.L., Dittrich, T.R., Döppner, T., Hinkel, D.E., Berzak Hopkins, L.F., Kline, J.L., Le Pape, S., Ma, T., MacPhee, A.G., Milovich, J.L., PaK, A., Park, H.-S., Patel, P.K., Remington, B.A., Salmonson, J.D., Springer, P.T. $\&$ Tommasini, R. (2014). Fuel gain exceeding unity in an inertially confined fusion implosion. Nature 506, 343-348.

Jungwirth, K., Cejnarova, A., Juha, L., Kralikova, B., Krasa, J., Krousky, E., Krupickova, P., Laska, L., Masek, K., Mocek, T., Pfeifer, M., Präg, A., Renner, O., Rohlena, K., Rus, B., Skala, J., Straka, P. \& Ullschmied, J. (2001). The prague asterix laser system phys. Plasmas 8, 2495-2501.

Kanngiesser, K.W., Huang, D.H. \& LiPs, H. (1994). Highvoltage direct current transmission - Systems and Planning. Siemens monographs Munich, EV HA 7.

Korn, G., Margarone, D. \& Picciotto, A. (2014). Boron-Proton Nuclear Fusion Enhancement Induced in Boron-doped Silicon Targets by Low-contrast Pulsed Lasers. IZEST ELI-NP Conf. Paris, 17-18 September 2014.

Kouhi, M., Ghoraneviss, M., Malekynia, B., Hora, H., Miley, G.H., SARI, A.H., AzIZI, N. \& RaZAVIPOURM, S.S. (2011). Resonance effect for strong increase of fusion gains at thermal compression for volume ignition of Hydrogen Boron-11. Laser Part. Beams 29, 125.
Krasa, J., Klier, D., Velyhan, A., Margarone, D., Krousky, E., Jungwirth, K., Skala, J., Pfeifer, M., Kravarik, J., Kubes, P., ReZAK, K. \& Ullschmied, J. (2013). Observation of repeitive bursts in emission of fast ions and and neutrons in subnanosecond laser-solid experiemnts. Laser Part. Beams 31, 395-401.

Labaune, C., Deprierraux, S., Goyon, S., Loisel, C., Yahia, G. \& RAFELSKI, J. (2013). Fusion reactions initiated by laser acceleratedparticle beams in laser produced plasmas. Nat. Commun. $\mathbf{4}$, 2506.

Lalousis, P. \& Hora, H. (1983). First direct electron and ion fluid computation of high electrostatic fields in dense inhomogeneous plasmas with subsequent nonlinear laser interaction. Laser Part. Beams 1, 283-304.

Lalousis, P., Hora, H. \& Moustaizis, S. (2014). Optimized boron fusion with magnetic trapping by laser driven shock initiation at nonlinear force driven ultrahigh acceleration. Laser Part. Beams 32, 409-411.

Lalousis, P., Hora, H., Eliezer, S., Martnez-Val, J.-M., MoustaizIs, S., Miley, G.H. \& Mourou, G. (2013). Shock mechanisms by ultrahigh laser accelerated plasma blocks in solid density targets for fusion. Phys. Lett. A 377, 885-888.

Lalousis, P., Moustaizis, S., Hora, H. \& Miley, G.H. (2015). Kilotesla magnetic assisted fast laser ignited Boron-11 hydrogen fusion with nonlinear force driven ultrahigh accelerated plasma blocks. J. Fusion Energy 34, 62-67.

Li, R.X., Liang, X.Y., Chu, Y.X., Gan, Z.B., Lu, H.H., Yu, L.H., YIN, D.J., Leng, Y.X., Lu, X.M., WANG, C. \& Xu, Z.Z. (2015). Progress towards a $10 \mathrm{PW}$ ultraintense laser facility: Demonstration of 5 PW high gain large aperture Ti:saphhire amplifier. $4^{\text {th }}$ Advanced Lasers and Photon Sources (ALPS'15), Yokohama, Japan April 22-24.

MaLeKynia, B. \& RaZIPUR, S.S. (2013). Internal propagation of fusion flame with shock strong of laser driven plasma block for advanced nuclear fuels ignition. Chin. Phys. B22, 055202.

Margarone, D., Picciotto, A., Velyhan, A., Krasa, J., Kucharik, M., Mangione, A., Szydlowsky, A., Malinowska, A., Bertuccio, G., Shi, Y., Crivellari, M., Ullschmied, J., Bellutti, P. \& Korn, G. (2015). Advanced scheme for high-yield laser driven nuclear reactions. Plasma Phys. Control. Fusion 57, 014030.

Mitchell, W. (2000). Keynote lecture at the ECLIM conference Schliersee/Germany.

Mourou, G., Barty, C.P.L. \& Perry, M.D. (1998). Ultrahighintensity lasers: physics of the extreme on a tabletop. Phys. Today 51, 22-30.

Mourou, G., Brocklesby, B., Tajima, T. \& Limpert, L. (2013). The future is fibre accelerators. Nat. Photonics 7, 258-261.

Moustaizis, S., Lalousis, P. \& Hora, H. (2013). A LIF scheme for HiPER application based on the combination of ultrahigh laser nonlinear force driven plasma blocks and the relativistic acceleration of ion blocks. In High Power, High Energy and Hig-intensity Laser Technology and Research using Extreme Light: Entering New Frontiers with Petawatt-class Lasers. (Hein, J., Korn, G. and Silva, L.O., Eds.). Proceedings of SPIE Vol. 8780, paper 878029/1-10.

Nevins, W.M. \& Swain, R. (2000). The thermonuclear fusion rate coefficients for p-11B reactions. Nucl. Fusion 40, 865.

Norreys, P.A., Fews, A.P. Beg, F.N., Bell, A.R., Dangor, A.E., Lee, P., Nelson, M.B., Schmidt, H., Tatarakis, M. \& Cable, M.D. (1998). Neutron production from picosecond laser 
irradiation of deuterated targets at intensities of $10^{19} \mathrm{~W} / \mathrm{cm}^{2}$ Plasma Phys. Control. Fusion 40, 175.

Oliphant, M.L.E., Harteck, P. \& Rutherford, L. (1934). 100 kilovolt discharges in deuterium plasmas. Proc. R. Soc. Lond. A 144, 692-714.

Oliphant, M.L.E. \& Rutherford, L. (1933). Experiments on the transmutations of elements by protons. Proc. R. Soc. Lond. A 141, 259-281.

PAterson, A. (2015). Latest developments on small modular reactors. Bull. R. Soc. NSW No. 385, 4.

Picciotto, A., Margarone, D., Velyhan, A., Bellini, P., Krasa, J., Szydlowski, A., Bertuccio, G., Shi, Y., Margarone, A., ProkuPeK, J., MalinowsKa, A., Krouski, E., Ullschmied, J., Laska, L., KUCHARIK, M. \& KoRN, G. (2014). Boron-proton nuclear-fusion enhancement induced in boron-doped silicon targets by lowcontrast pulsed laser. Phys. Rev. X 4, 031030.

Rostoker, N., Binderbauer, M.W. \& Monkhorst, H.J. (1997). Colliding beam fusion reactor. Science 278, 1419-1422.

Santos, J.J., Bailly-Gandvoux, M., Giuffrida, L., ForesterColleoni, P., Fuijoka, S., Zang, Z., Korneev, Ph, Boullaud, R., Dorand, S., Batani, D., Chervrrot, M., Cross, J., Crowston, R., Dubois, J.-L., Gazave, J., Greofri, G., D’Humieres, E., Hulin, S., Ishihara, K., Kojima, S., Loyez, E., Marques, J.-R., Nicolai, Ph., Peyrusse, O., Poye, A., Raffestin, D., Ribolzi, J., Roth, M., Schaumann, G., Serred, F., Tikhonchuk, V.T.,
Vacar, Ph. \& Woolsley, N. (2015). Laser-driven platform for generation and characterization of strong quasi-staticmagnetic fields. Phys. Plasmas arXiv 1503.00247/1-16.

Sauerbrey, R. (1996). Acceleration of femtosecond laser produced plasmas. Phys. Plasmas 3, 4712-4716.

Strickland, D. \& Mourou, G. (1985). Compression of amplified chirped optical pulses. Opt. Commun. 56, 219-222.

TabaK, M., Hammer, J., Glinsky, M.N., KRUER, W.L., Wilks, S.C., Woodworth, J., Campbell, E.M., Perry, M.D. \& Mason, R.J. (1994). Ignition and high gain with ultrapowerful lasers. Phys. Plasmas 1, 1626-1634.

TAhIR, N.A. \& Hoffmann, D.H.H. (1997). Development of advanced inertial fusion targets. Laser Part. Beams 15, 575-587.

Weaver, T., Zimmerman, G. \& Wood, L. (1973). Exotic CTR Fuels: Non-thermal Effects and Laser Fusion Applications. Lawrence Livermore National Laboratory Report UCRL 74938.

Yu, W., Xu, H., He, F., Yu, M.Y., Ishoguro, S., Zhang, J. \& Wong, A.Y. (2005). Direct acceleration of solid-density plasma bunch by ultraintense laser. Phys. Rev. E 72, 046401.

Zhang, M., He, J.T., Chen, D.B., Li, Z.H., Zhang, Y., Wang, L., Feng, B.L., Zhang, D.F., Tang, X.W. \& Zhang, J. (1998). Effects of a prepulse on $\gamma$-ray radiation produced by a femtosecond laser with only 5-mJ energy. Phys. Rev. E 57, 3746-3748. 